\title{
Differences in age-related effects on brain volume in Down syndrome as compared to Williams syndrome and typical development
}

Mary Ellen I Koran ${ }^{1,2}$, Timothy J Hohman ${ }^{1}$, Courtney M Edwards ${ }^{1,3}$, Jennifer N Vega ${ }^{1,4}$, Jennifer R Pryweller, Laura E Slosky' ${ }^{1}$, Genea Crockett ${ }^{1}$, Lynette Villa de Rey' ${ }^{1}$, Shashwath A Meda' ${ }^{1}$, Nathan Dankner ${ }^{6,8}$, Suzanne N Avery ${ }^{4,7}$, Jennifer U Blackford ${ }^{7,8}$, Elisabeth M Dykens ${ }^{7,8,9}$ and Tricia A Thornton-Wells $s^{1,8,10^{*}}$

\begin{abstract}
Background: Individuals with Down Syndrome (DS) are reported to experience early onset of brain aging. However, it is not well understood how pre-existing neurodevelopmental effects versus neurodegenerative processes might be contributing to the observed pattern of brain atrophy in younger adults with DS. The aims of the current study were to: (1) to confirm previous findings of age-related changes in DS compared to adults with typical development (TD), (2) to test for an effect of these age-related changes in a second neurodevelopmental disorder, Williams syndrome (WS), and (3) to identify a pattern of regional age-related effects that are unique to DS

Methods: High-resolution T1-weighted MRI of the brains of subjects with DS, WS, and TD controls were segmented, and estimates of regional brain volume were derived using FreeSurfer. A general linear model was employed to test for age-related effects on volume between groups. Secondary analyses in the DS group explored the relationship between brain volume and neuropsychological tests and APOE.

Results: Consistent with previous findings, the DS group showed significantly greater age-related effects relative to TD controls in total gray matter and in regions of the orbitofrontal cortex and the parietal cortex. Individuals with DS also showed significantly greater age-related effects on volume of the left and right inferior lateral ventricles (LILV and RILV, respectively). There were no significant differences in age-related effects on volume when comparing the WS and TD groups. In the DS group, cognitive tests scores measuring signs of dementia and APOE $\varepsilon 4$ carrier status were associated with LILV and RILV volume.

Conclusions: Individuals with DS demonstrated a unique pattern of age-related effects on gray matter and ventricular volume, the latter of which was associated with dementia rating scores in the DS group. Results may indicate that early onset of brain aging in DS is primarily due to DS-specific neurodegenerative processes, as opposed to general atypical neurodevelopment.
\end{abstract}

Keywords: Down syndrome, Williams syndrome, Neurodevelopmental disorder, Brain volume, APOE, MRI, Accelerated aging, Neuroimaging genetics, Alzheimer's disease

\footnotetext{
*Correspondence: t.thornton-wells@vanderbilt.edu

${ }^{1}$ Center for Human Genetics and Research, Department of Molecular

Physiology \& Biophysics, Vanderbilt University School of Medicine,

37232-0700, 519 Light Hall, Nashville, TN, USA

${ }^{8}$ Vanderbilt Kennedy Center for Research on Human Development,

Vanderbilt University, Nashville, TN, USA

Full list of author information is available at the end of the article
} 


\section{Background}

It has been suggested that individuals with Down syndrome (DS) experience early onset, or perhaps accelerated, brain aging as evidenced by significant age-related reduction in brain volume, but to date, it is not known whether this is an effect of the accumulated neurotoxic pathology evident in DS or a pre-existing feature of atypical neurodevelopment [1]. In the current study, we aimed to dissociate the contribution of neurodevelopment versus neurodegeneration in regional brain volume in DS. We compared a wide age range of adults with DS to adults with a different neurodevelopmental disorder, Williams syndrome (WS), and we also compared both neurodevelopmental disorders to a typically developing (TD) control group.

\section{Etiology and presentation of Down syndrome and Williams syndrome}

DS is a neurodevelopmental disorder caused by the presence of three copies of chromosome 21 (trisomy 21). It occurs in one in every 691 live births in the US [2] and is the most common genetic cause of intellectual disability [3,4]. The degree of cognitive impairment associated with DS ranges from mild to severe, with the mean IQ of 50, or moderate intellectual disability [4]. Individuals with DS exhibit deficits in language, verbal short-term memory, and explicit long-term memory; whereas visuospatial short-term memory, associative learning, and implicit memory are relatively preserved [5]. Advances in the treatment of medical comorbidities, such as heart defects and digestive malformations, have resulted in dramatic improvements in life expectancy for individuals with DS living in the US, rising from nine years in the early-twentieth century [6] to nearly 60 years in 2010 [7]. Although individuals with DS present with a unique cognitive and behavioral profile, they do share some basic characteristics with individuals who have WS.

WS is a neurodevelopmental disorder caused by the hemizygous deletion of 26 to 28 genes on chromosome 7 [8]. The prevalence of WS is one in every 7,500 live births [9]. As with DS, WS is associated with intellectual disability. The average IQ for individuals with WS is approximately 50 to 60 , indicating mild to moderate intellectual disability $[10,11]$. The WS cognitive profile is characterized by deficits in visuospatial and implicit memory as well as strengths in language, verbal shortterm memory, face and object recognition, and music processing skills [12-16]. In addition, individuals with WS often demonstrate increased non-social anxiety and phobias, paired with hypersociability and heightened empathy [17-19]. Similar to individuals with DS, persons with WS have experienced a significant increase in mean life expectancy following advances in treatment for medical comorbidities, particularly cardiac defects.
There is very little literature on aging or life expectancy in WS, but there are documented cases of persons with WS who lived to be 70 years old [20].

\section{Brain morphometry in Down syndrome and Williams syndrome}

Children and young adults with DS or WS have an overall smaller brain volume compared to TD individuals of similar age [21]; however the specific brain areas that show significant volumetric differences compared to TD are distinct for each of the neurodevelopmental disorders. Individuals with DS have smaller frontal, amygdalar, and cerebellar volumes compared to TDs; whereas individuals with WS have smaller midbrain, thalamic, basal ganglia, and occipital and superior parietal lobe volumes compared to age-matched TDs $[22,23]$. It is important to also note that individuals with DS have an increase in parahippocampal volume and relatively preserved lenticular nuclei, basal ganglia, and occipital lobe volumes $[4,5,24]$. Young adults with WS have relatively preserved frontal lobe, anterior cingulate, superior temporal and fusiform gyrus, amygdalar, and cerebellar volumes compared to TDs [5,22,24].

In addition to the pre-existing smaller volumes of frontal, amygdalar and cerebellar structures, older adults with DS ( $>50$ years of age) have been shown to also have smaller whole prefrontal, posterior cingulate, hippocampal, and parahippocampal volumes when compared to age-matched TD adults [5]. Studies have shown that as individuals with DS age, they exhibit a similar pattern of neurodegeneration to that seen in the early stages of Alzheimer's disease in the general population, in which the earliest neuropathological changes present in the medial-temporal lobe and progress to neocortex and subcortical regions [25]. However, in individuals with DS these neuropathological changes occur at a much younger age compared to the general population, which has been attributed to early onset, or perhaps accelerated, brain aging [1].

At present, very few studies have assessed changes in brain morphology in older adults with WS [26]. Studies have shown an overall $15 \%$ smaller brain volume in adults with WS between 19 and 52 years of age compared to age-matched TD controls [20,21]; however, they found no difference in the magnitude of this finding between older individuals with WS and a group of younger persons with WS, suggesting the effect might not be age-related [20].

\section{Study aims}

The aims of the current study were to: (1) confirm previous findings of age-related brain changes in DS versus TD, (2) document any age-related differences in brain volume seen in WS versus TD, and (3) test for age-related effects that are unique to DS. If the changes seen in DS are primarily due to DS-specific neurodegenerative processes, 
then we would hypothesize that in the DS group, the agerelated effects would be greater than those in the TD and WS groups. If, however, these changes are instead associated with a non-specific vulnerability due to atypical neurodevelopment, then we would hypothesize that both DS and WS groups would show greater age-related differences as compared to TD adults than they would compared to each other.

\section{Methods}

\section{Study participants}

The current study included 14 DS adults (7 males; mean age 39; age range: 19 to 63$), 41$ WS adults (24 males; mean age 26, age range: 16 to 58 ), and 82 TD adults ( 40 males; mean age 36 , age range: 18 to 90 ). Adults with DS or TD were recruited using flyers and website postings with Institutional Review Board-approved language targeting adults over 18 years of age. For adults with DS, we further recruited from local and regional educational centers for individuals with intellectual disabilities, community-based assisted living centers, caregiver support groups, and employment assistance programs. Participants with WS were recruited through the annual Academy of Country Music Lifting Lives Music Camp, which is organized by the Vanderbilt Kennedy Center for Research on Human Development. All participants with WS or DS exhibited the physical, cognitive, and behavioral profile of WS and DS, respectively, and they previously had received a clinical diagnosis of the disease. Adults with typical neurodevelopment were ascertained either as age-matched controls for study participants with WS or as healthy older adults who served as controls for a general population study of age-related cognitive impairment. The three groups were tested for differences in age and sex, using an independent samples $t$-test and a chi-square test, respectively. Demographic characteristics along with their corresponding statistical values are detailed in Table 1 .
Participants with TD and caregivers of individuals with DS or WS gave informed consent, while participants with DS or WS gave informed assent for this study. All study procedures were approved by the Vanderbilt University Institutional Review Board.

\section{Magnetic resonance imaging acquisition}

Adults participated in a magnetic resonance imaging (MRI) scan in a Philips Achieva 3-Tesla scanner (Philips Medical Systems, Inc., Best, Netherlands) using an eightchannel SENSE head coil, housed in the Vanderbilt University Institute of Imaging Science (Nashville, TN, USA). High-resolution three-dimensional anatomical T1-weighted MRI images were acquired using a turbo field echo sequence with full brain coverage and the following parameters: field of view $=256 \times 256 \mathrm{~mm}^{2}$; in plane voxel resolution $=1 \times 1 \mathrm{~mm}^{2}$; repetition time $=8.9 \mathrm{~ms}$; echo time $=4.6 \mathrm{~ms}$; flip angle $=8^{\circ}$; slice thickness $=1 \mathrm{~mm}$ and 170 slices with no slice gap.

\section{Neuroimaging analysis}

To parcellate the brain into cortical and subcortical tissue classes and derive quantitative estimates of brain volume, we used an automated, non-biased atlas-based Bayesian segmentation procedure, applied in FreeSurfer v.5.0 (http://surfer.nmr.mgh.harvard.edu/) [27]. FreeSurfer preprocessing for volumetric T1-weighted images included: brain extraction and removal of non-brain tissue using a hybrid watershed/surface deformation procedure [28]; automated spatial transformation and white matter segmentation of subcortical volumetric structures [29]; intensity normalization, tessellation of gray matter/white matter boundary and automated topology correction [30]; and surface deformation following intensity gradients to optimally place gray matter/white matter and gray matter/cerebrospinal fluid borders at the location where the greatest shift in intensity defines the transition to the other tissue class [27]. Image outputs from each

Table 1 Sample demographics for participants with Down syndrome (DS), Williams syndrome (WS), and typically developing (TD) controls

\begin{tabular}{|c|c|c|c|c|c|c|c|c|c|c|c|c|c|c|c|}
\hline & & & & & & & & & & \multicolumn{6}{|c|}{ Group contrasts } \\
\hline & \multicolumn{3}{|c|}{$D S(N=14)$} & \multicolumn{3}{|c|}{ TD $(\mathrm{N}=82)$} & \multicolumn{3}{|c|}{ WS $(N=41)$} & & & \multicolumn{2}{|c|}{ DS versus WS versus TD } & & \\
\hline & $\mathrm{N}$ & $\%$ & & $\mathrm{~N}$ & $\%$ & & $\mathrm{~N}$ & $\%$ & & & & $x^{2}$ & $P$-value & & \\
\hline \multirow[t]{3}{*}{ Male sex } & 7 & 50 & & 40 & 49 & & 24 & 59 & & & & 1.89 & 0.39 & & \\
\hline & & & & & & & & & & DS v & ersus TD & WS & us TD & DS v & ersus WS \\
\hline & Range & Mean & SD & Range & Mean & SD & Range & Mean & SD & $t$ & $P$-value & $t$ & $P$-value & $t$ & $P$-value \\
\hline \multirow[t]{2}{*}{ Age (years) } & 19 to 63 & 39 & 13 & 18 to 90 & 36 & 18 & 16 to 58 & 26 & 8 & 0.61 & 0.54 & -3.44 & $1.0 \mathrm{E}-03$ & 4.31 & $7.1 \mathrm{E}-05$ \\
\hline & Range & Median & SD & & & & & & & & & & & & \\
\hline DLD-SCS & 0 to 21 & 0.5 & 8 & & & & & & & & & & & & \\
\hline DLD-SOS & 0 to 18 & 5 & 5 & & & & & & & & & & & & \\
\hline
\end{tabular}

$\mathrm{SD}=$ standard deviation, $\mathrm{N}=$ number or count, $\%=$ percentage of subjects in the group corresponding to the preceding count, $t=t$-statistic, and $\mathrm{X}^{2}=$ chi-square test of independence statistic. 
stage of FreeSurfer processing were visually inspected independently by three imaging analysts (CME, MEK). Only images that passed quality control by both analysts were used; seven DS, five WS, and five TD adults were not included in the analysis because of failure to pass quality control, leaving a total of 14,58 and 81 adults in each category, respectively. Quantitative estimates of volume were derived in a large set of spatially distinct region of interests (ROIs) that covered the entire brain, as specified in the Desikan atlas [31]. This atlas includes parcellations of gray and white matter and segmentations of subcortical gray matter, and also includes summary volumes (that is total cortex volume). Parcellations of the gray and white matter and segmentations of subcortical gray matter were included, along with two summary measurements of total gray and total white matter (see Additional file 1: Tables S1a and b for complete list of ROIs included and those excluded, respectively). Total intracranial volume (ICV) was also estimated in FreeSurfer, and all ROI measures were normalized to ICV for subsequent analyses.

\section{Genotyping}

Adults with DS had blood drawn for DNA, which was directly genotyped for $A P O E$ using pre-made TaqMan SNP genotyping assays from Applied Biosystems (ABI; Foster City, CA, USA). Negative controls (no template) and positive controls (DNA samples with known genotypes from Coriell Institute for Medical Research, Camden, NJ, USA) were included on the plate for assay validation. Since genotyping was performed in a research laboratory that is not CLIA-certified, genotyping results were not returned to patients or their clinicians.

\section{Cognitive testing}

For all participants with Down syndrome, we conducted a comprehensive battery of cognitive and neuropsychological tests (see Additional file 1: Table S2), including the Dementia Questionnaire for People with Learning Disabilities (DLD; Harcourt Assessment, Amsterdam, Netherlands, 2006) [32]. Although there is no 'gold standard' for assessing dementia in individuals with DS, studies have shown that the DLD is useful in the differential diagnosis of dementia [33,34]. The DLD is a 50 -item questionnaire that consists of eight subtests (short-term memory, long-term memory, and spatial and temporal orientation, speech, practical skills, mood, activity and interest, and behavioral disturbance) that assess both cognition and social skills. For each item, a score of 0 indicates no deficit, 1 indicates moderate deficit, and 2 indicates severe deficit. The sum of cognitive scores (SCS) is calculated from the short-term memory, longterm memory, spatial and temporal orientation subtests, and the range of possible scores for the DLD-SCS is 0 to
44. The sum of social scores (SOS) is calculated from the speech, practical skills, mood, activity, and interest and behavioral disturbance subtests, and the range of possible scores for the DLD-SOS is 0 to 60. Higher scores on each subtest indicate greater impairment. A masters-level study coordinator with training and experience in cognitive and neuropsychological assessment administered the DLD. Complete results of neuropsychological testing for the DS participants, as well as $A P O E \varepsilon 4$ carrier status, can be seen in Additional file 1: Table S2. We also note that none of the participants with DS were taking anti-depressants, antipsychotics or cholinesterase-inhibitors, which could confound cognitive testing results, although one person was taking the anti-convulsant 'Lamictal', which is a sodium channel blocker.

\section{Statistical analysis}

Our first two aims were to (1) confirm previous findings of early age-related changes in DS compared to TD and (2) test for a comparable effect in WS relative to TD. In order to approach these aims, we implemented a general linear model in R (http://www.R-project.org) across 103 separate regions of interest:

$$
\begin{aligned}
& (\mathrm{ICV} \text {-corrected volume of each ROI }= \\
& \quad \text { Group }+ \text { Age }+ \text { Sex }+(\text { Group } \times \text { Age }))
\end{aligned}
$$

Group status was dummy-coded in the regression model with TD set as the reference category. Thus, our model included two group main effects (DS as 0 or 1 and WS as 0 or 1 ) and two interaction terms: age $\times$ DS and age $\times$ WS which statistically compare the age-related slopes of regional volume between the respective diagnostic category and TD controls. This method and a three category method in ANCOVA are statistically equivalent [35]. However, in the linear regression method used here, the $t$-tests based on dummy-coded variables directly test the alternative hypothesis that the coded group differs from the reference group (in this case, TD controls), which aids in the interpretation of results.

Sex was coded as a binary discrete variable (male as 0 ; female as 1). A Bonferroni corrected significance threshold of $P<4.85 \times 10^{-4}$ was applied to the interaction terms in order to correct for the 103 ROIs tested (see Additional file 1: Table S2a for list of ROIs tested). A post hoc analysis was conducted to determine the sensitivity of results due to outliers. Outliers were defined as adults with ICV-corrected total gray matter volume outside of the grand mean \pm two standard deviations (calculated in SPSS, www.ibm.com/software/analytics/spss/).

A final aim was to test for age-related effects unique to DS. The same general linear model was used (ICVcorrected volume of each $\mathrm{ROI}=$ Group + Age + Sex + 


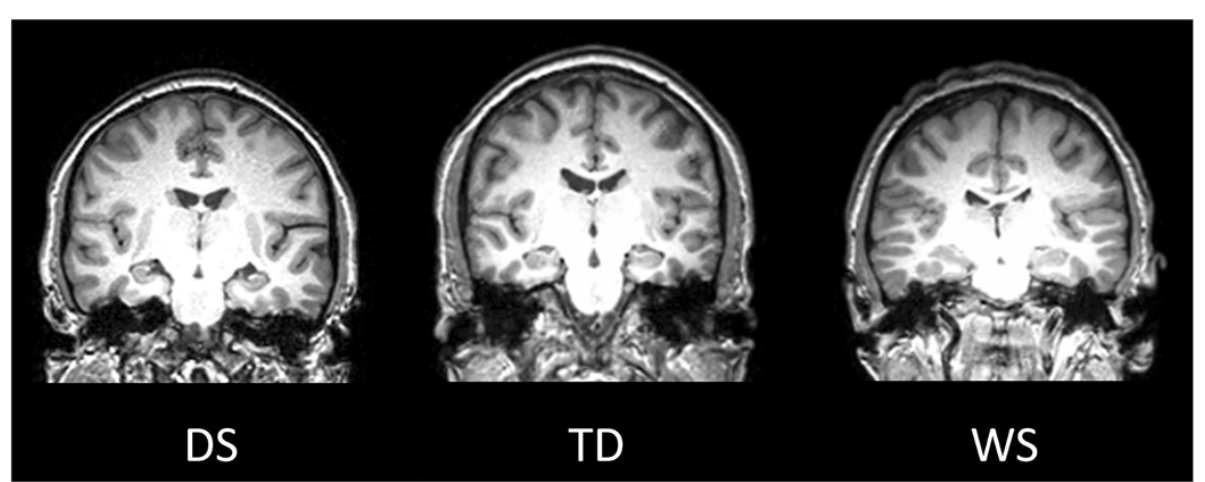

Figure 1 Representative T1-weighted MRI for each group (Down syndrome (DS), typically developing (TD), and Williams syndrome (WS)). The subjects are 35, 36, and 38 years old respectively. The inferior lateral ventricles can be seen as hypo-intense spaces around the hippocampus in the temporal lobes.

$($ Group $\times$ Age $)$ ), but we only compared DS and WS subjects (WS $=0$ and $\mathrm{DS}=1$ ). A Bonferroni correction was applied to determine the significant interaction terms, and again a post hoc analysis was performed after removing statistical outliers.

\section{Secondary exploratory analyses}

In order to further explore age-related volume that is specific to DS, we performed linear regression to test for an association between brain volume and other cognitive and genetic risk factors. Since DLD and genotyping data were only collected for participants with DS, analyses were restricted to the DS group. To control for Type I error, we restricted our analysis to brain regions that showed significant age-related effects, which were stronger in DS relative to TD and WS. Predictors included age, sex, and the variable of interest. For each ROI, we tested for an association with DLD-SOS or DLD-SCS as the continuous variable of interest. We report the $t$-statistic for the variable of interest (DLD-SOS or DLD-SCS), along with its unadjusted $P$-value, and we report the change in $\mathrm{R}^{2}$ comparing the full model with the variable of interest to the reduced model with only age and sex.

Next we performed an exploratory analysis to determine the relationship between $A P O E$ \&4 carrier status and age-related volume effects in DS. The absence/presence of $A P O E \& 4$ alleles was coded as $0 / 1$, respectively. We report the unadjusted $P$-value of the $A P O E$ term and the change in $\mathrm{R}^{2}$ for the full model with $A P O E$ to the reduced model with only age and sex as predictors.

\section{Results}

Example T1-weighted MR images for each group can be seen in Figure 1. Our first analysis aimed to (1) replicate previous findings of age-related effects on brain in DS and (2) test for similar effects in WS. Consistent with previous findings, the DS group showed significantly greater age-related effects on gray matter volume relative to TD controls in the regions of the orbitofrontal cortex (the left pars orbitalis) and the parietal cortex (the left superior parietal lobe, the left inferior parietal lobe and the right post central gyrus; Table 2, Additional file 1: Table S3; Figure 2). Individuals with DS also showed significant age-related effects on volume of the left and right inferior lateral ventricles (LILV and RILV, respectively, Figure 3). In contrast, there were no significant differences in age-related volume between WS and TD controls. The summary measurements of total white matter and total gray matter volumes were also analyzed, and the DS group showed greater age-related effects on total gray matter volume (unadjusted $P=.007$ ) while there was no significant effect in the total white matter volume measure $(P=.055)$.

Our second analysis compared age-related volume in DS to age-related volume in WS (Table 3; Additional file 1: Table S4). The DS group showed a stronger relationship between age and volume for the left and right lateral ventricles relative to WS, and this difference remained

Table 2 Brain regions where the relationship between age and volume was significantly different between participants with Down syndrome (DS) versus typical development (TD)

\begin{tabular}{lccc}
\hline Region of interest & \multicolumn{3}{c}{ Group (DS versus TD) $\times$ Age Interaction } \\
\cline { 2 - 4 } & $\boldsymbol{t}$ & $\boldsymbol{P}$-value & Difference in $\mathbf{R}^{\mathbf{2}}$ \\
\hline LILV & 4.31 & $3.25 \mathrm{E}-05$ & 0.09 \\
RILV & 4.05 & $9.03 \mathrm{E}-05$ & 0.10 \\
Left superior parietal & -4.02 & $1.01 \mathrm{E}-04$ & 0.08 \\
Left inferior parietal & -3.97 & $1.23 \mathrm{E}-04$ & 0.08 \\
Left pars orbitalis & -3.82 & $2.11 \mathrm{E}-04$ & 0.07 \\
Right post central gyrus & -3.67 & $3.58 \mathrm{E}-04$ & 0.07 \\
\hline
\end{tabular}

Difference in $\mathrm{R}^{2}$ is reported for full model that included age, sex, group and groupxage, versus the reduced model that included age, sex, and group. 


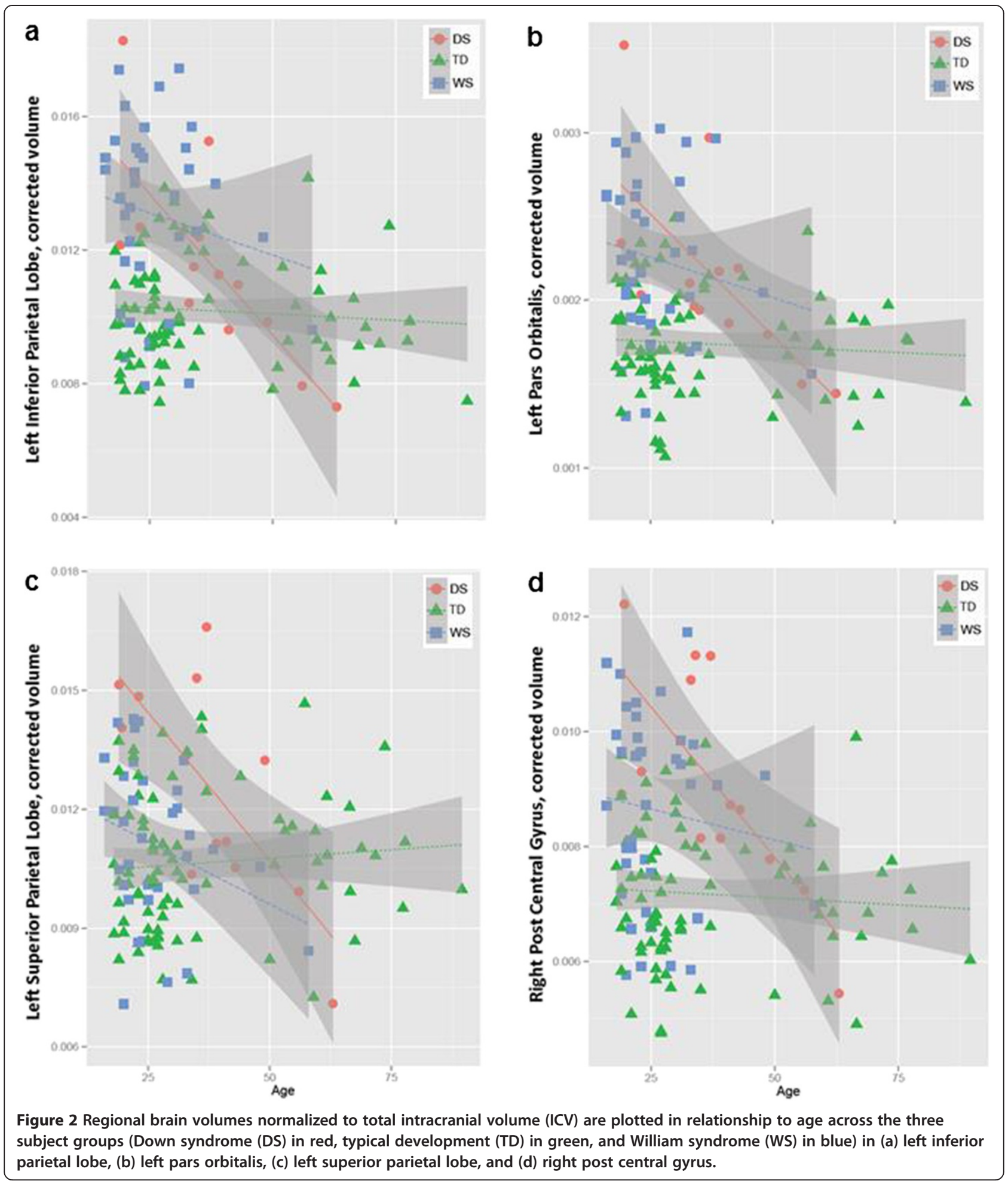

significant after correcting for multiple comparisons. Plots of age versus volume for all significant ROIs are presented in Figures 2 and 3.

To investigate whether outliers were driving the observed effects, we removed the participants whose ICV- corrected total gray matter volume was outside of two standard deviations of the grand mean, and we repeated our analyses. One DS subject and four WS adults were removed, but no outliers were identified for the TD group. For the first regression analysis, the interaction 

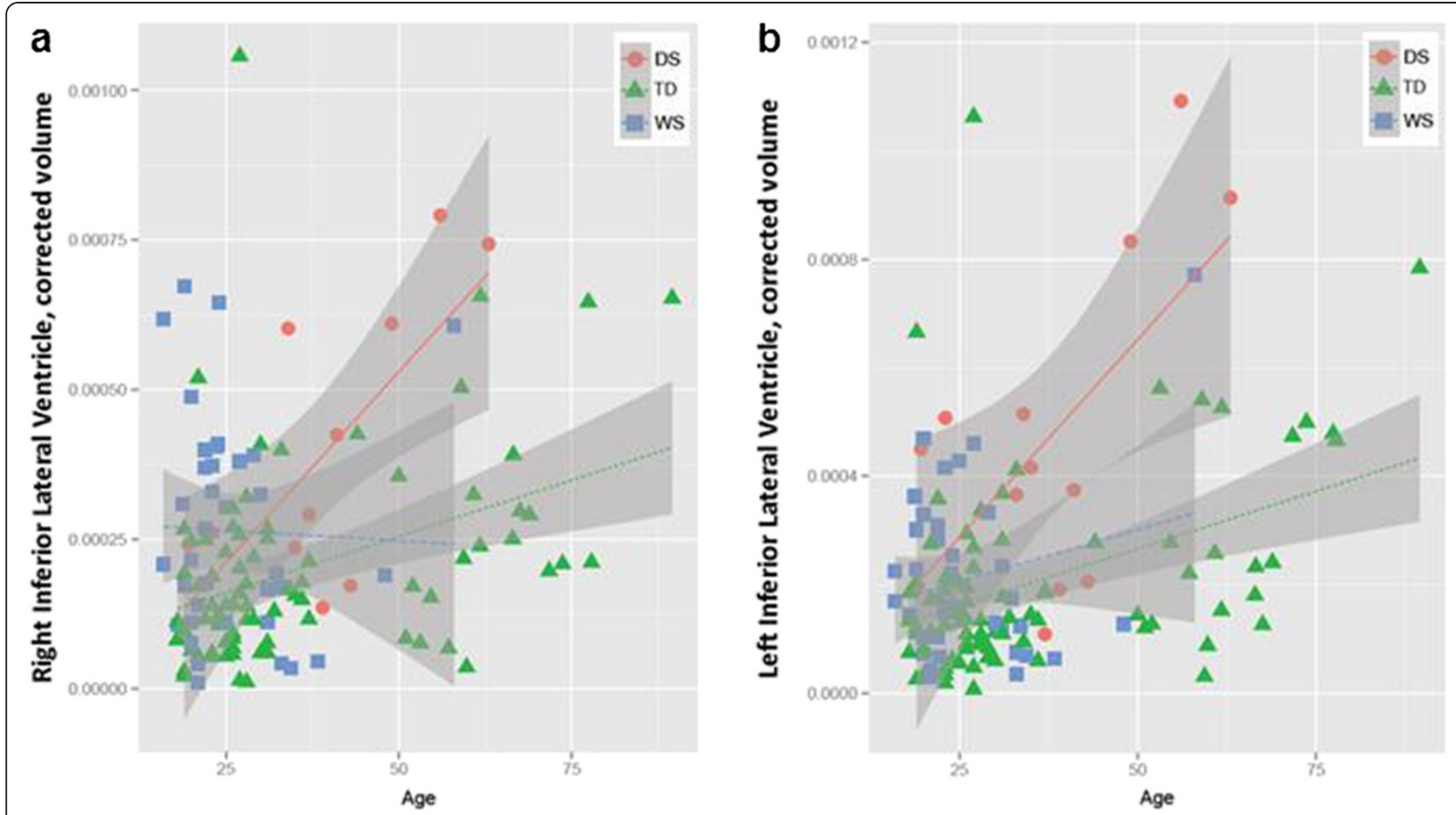

Figure 3 Regional brain volumes normalized to total intracranial volume (ICV) are plotted in relationship to age across the three subject groups (Down syndrome (DS) in red, typical development (TD) in green, and William syndrome (WS) in blue) in: (a) left inferior lateral ventricle (LILV) and (b) right inferior lateral ventricle (RILV).

model term Group (DS versus TD)x Age, comparing the relationship between volume and age between DS and TD groups, remained significant after Bonferroni correction for the left superior parietal lobe, and in all other ROIs found significant in primary analyses, the interaction term was nominally significant at an unadjusted $P<.05$. For the second regression analysis, the interaction model term comparing age-related effects in volume between the DS and WS groups remained significant after Bonferroni correction for the right lateral ventricle, and for the left lateral ventricle, the interaction term was nominally significant at an unadjusted $P<.05$. No additional ROIs reached Bonferroni significance after outliers were removed in either analysis. These results are presented in Additional file 1: Tables S5a and S5b and Additional file 1: Figure S1.

Table 3 Brain regions where the relationship between age and volume was significantly different between participants with Down syndrome (DS) versus Williams syndrome (WS)

\begin{tabular}{lccc}
\hline Region of interest & \multicolumn{3}{c}{ Group (DS versus WS) $\times$ Age Interaction Term } \\
\cline { 2 - 4 } & $\boldsymbol{t}$ & $\boldsymbol{P}$-value & Difference in $\mathbf{R}^{\mathbf{2}}$ \\
\hline Right lateral ventricle & 4.42 & $8.64 \mathrm{E}-05$ & 0.14 \\
Left lateral ventricle & 3.75 & $1.35 \mathrm{E}-04$ & 0.12 \\
\hline
\end{tabular}

The right lateral ventricle association was significant after Bonferroni correction.
In secondary analyses, we explored the relationships between brain volume in the eight significant ROIs reported above and the DLD-SOS score, the DLD-SCS score, and $A P O E$ status in DS adults using linear regression. Full results are presented in Table 4. DLD-SCS and -SOS scores were significantly intercorrelated $\left(\mathrm{r}^{2}=.770, P=.002\right)$ and explained similarly high rates of variability in regional brain volume, beyond that explained by the reduced model including age and sex. DLS-SCS scores explained 21 and $25 \%$ of the variance in LILV and RILV volume, respectively ( $P=.042$ and $P=.031$; Additional file 1: Figure S2). DLD-SOS scores explained $38 \%$ of variance of both the LILV and RILV volumes $(P=.001$ and $P=.003$; Additional file 1: Figure S3). DLD-SOS was also nominally associated with the overall right ventricular volume $(P=.029)$ and explained $15 \%$ of right ventricular volume variance. $A P O E$ explained $22 \%$ of the variance in LILV and RILV volume $(P=.026$ and .033; Additional file 1: Figure S4).

Thus, in summary, all three variables were nominally associated with LILV and RILV volume $(P<.05)$, and the association between DLD-SOS and RILV volume was significant after Bonferroni correction. However, after the one DS outlier was removed, only the relationships between DLD-SCS and RILV and between DLD-SOS and LILV remained even nominally significant $(P=.037$ and .009 , respectively). Notably, the removed subject was one of three adults who had an APOE \&4 allele. 
Table 4 Relationship between Volume and Dementia Questionnaire for People with Learning Disabilities (DLD) sum of cognitive scores (SCS) and sum of social scores (SOS) or APOE in brain regions showing a correlation with age in Down syndrome participants

\begin{tabular}{|c|c|c|c|c|c|c|c|c|c|}
\hline \multirow[t]{2}{*}{ Region of interest } & \multicolumn{3}{|c|}{ DLD-SCS } & \multicolumn{3}{|c|}{ DLD-SOS } & \multicolumn{3}{|c|}{ APOE } \\
\hline & $t$ & $P$-value & Difference in $\mathrm{R}^{2}$ & $t$ & $P$-value & Difference in $\mathrm{R}^{2}$ & $t$ & $P$-value & Difference in $\mathrm{R}^{2}$ \\
\hline LILV & 2.37 & 0.042 & 0.21 & 4.72 & 0.001 & 0.38 & 2.61 & 0.026 & 0.22 \\
\hline RILV & 2.56 & 0.031 & 0.25 & 4.01 & 0.003 & 0.38 & 2.47 & 0.033 & 0.22 \\
\hline Left superior parietal & -1.32 & 0.219 & 0.06 & 0.41 & 0.693 & 0.01 & -1.06 & 0.315 & 0.04 \\
\hline Left pars orbitalis & -1.66 & 0.131 & 0.08 & -1.32 & 0.220 & 0.06 & -1.23 & 0.247 & 0.10 \\
\hline Left inferior parietal & -1.21 & 0.256 & 0.07 & -1.59 & 0.146 & 0.10 & -1.87 & 0.091 & 0.06 \\
\hline Right post central gyrus & -0.54 & 0.604 & 0.01 & -1.11 & 0.294 & 0.05 & 0.05 & 0.961 & 0.00 \\
\hline Left ventricle & 0.57 & 0.582 & 0.01 & 2.06 & 0.069 & 0.13 & 1.45 & 0.178 & 0.07 \\
\hline Right ventricle & 0.91 & 0.386 & 0.03 & 2.59 & 0.029 & 0.15 & 1.78 & 0.105 & 0.08 \\
\hline
\end{tabular}

Italicized $P$-values indicate nominal association at uncorrected $P<.05$. The Difference in $\mathrm{R}^{2}$ values indicates the difference in the variability in the region of interest (ROI) volume explained by the full model that included the predictor of interest (DLD-SCS, DLD-SOS, or APOE), compared to that explained by the reduced model with only age and sex as predictors.

\section{Discussion}

The first aim of the current study was to confirm previous findings of age-related brain changes in DS [1]. In previous studies, adults with DS had significantly stronger relationships between age and volume in the frontal, parietal, and temporal lobes, and the lateral ventricles [1]. We replicated these findings in the frontal lobe (specifically, the left pars orbitalis gyri frontalis inferioris), the parietal lobe (specifically, the left superior and left inferior parietal cortices and the right post central gyrus), and in the lateral ventricles (specifically, the LILV and RILV).

Interestingly, atrophy in these gray matter regions and dilation of the ventricles may be explained by the link between DS and Alzheimer's disease (AD). Individuals with DS are at a greatly increased risk of developing AD, with up to 70 percent developing dementia by the age of 70 [36]. In fact, adults with DS account for up to $60 \%$ of individuals with developmental disabilities who exhibit signs of $\mathrm{AD}$ [37]. The risk for $\mathrm{AD}$ in $\mathrm{DS}$ is primarily related to triplication of the amyloid precursor protein $(A P P)$, which is on chromosome 21 [38]. However, one's genotype at the apolipoprotein $\mathrm{E}(A P O E)$ gene, whose protein product is involved in the processing of amyloid beta isoforms, has also been shown to modulate risk for developing AD in the DS population $[39,40]$. Postmortem studies have revealed that plaque load in adults with DS increases with age: by 40 years of age, nearly all individuals with DS have amyloid beta plaques in the brain [41], and this characteristic feature of AD has neurotoxic effects that can lead to neurodegeneration and loss in brain volume [42]. More specifically, both the frontal and parietal lobes have shown increased amyloid load as measured by positron emission tomography (PET) in participants with DS [43]. Therefore, the age-related effects detected in this study may be due to the early-onset of neurotoxic effects related to increases in amyloid load in adults with DS.
Age-related volume in DS relative to WS adults was significant in the left and right total lateral ventricles, even after Bonferroni correction for multiple comparisons, and there was a stronger relationship between age and volume in the inferior lateral ventricles in the DS group compared to the TD group. These results are particularly interesting given the high prevalence of $\mathrm{AD}$ in $\mathrm{DS}$ and the association between ventricular dilation and AD. Volume of the lateral ventricles has repeatedly shown a relationship to AD status and disease progression in the general population [44-47]. The lateral ventricles normally dilate over time with age, as brain tissue volume decreases, but in patients with mild cognitive impairment (MCI) or AD, the rate of ventricular dilation is much greater than in the general aging population [48]. The inferior lateral ventricles are surrounded by subcortical gray matter structures, and these structures, particularly the hippocampus, entorhinal cortex, and amygdala, accumulate amyloid plaques and exhibit atrophy in AD $[48,49]$ and DS [50-54]. Since ventricular dilation is cumulatively and inversely reflective of atrophy of these surrounding structures [55], and since we found this strong relationship between age and ventricular volume was not present in WS, these results may be reflective of the neurodegenerative effects of AD pathology on the structures surrounding the ventricles.

We also investigated whether, similar to the DS group, the WS group experienced greater age-related effects compared to the TD group, but we did not find evidence to support this hypothesis. This is in line with the one previously reported finding of aging WS adults [20]. However, this may be due to the difference in age ranges between the WS and TD groups (Table 1), and further investigation of age-related volume in WS in a larger study, ideally with longitudinal data, is warranted.

As a secondary exploratory analysis in the DS cohort only, regions with significant age-related changes (in DS 
versus TD and DS versus WS) were evaluated for association with DLD-SOS, DLD-SCS and APOE \&4 status. We observed a strong relationship between ILV volume and both the cognitive and social scores on the DLD, a test that measures dementia-related impairments in the DS population, though only the relationship between RILV and DLDSCS and between LILV and DLD-SOS remained nominally significant after the one DS outlier was removed. While we have collected data from DS study participants using a comprehensive battery of tests, we do not have the clinical expertise and have not sought consensus from clinical experts to determine clinical dementia status. Instead, we have used the DLD scores as a quantitative proxy measure of behavioral symptoms related to dementia status. Previous studies have shown that individuals with DS have decreased regional brain volumes with onset of dementia $[50,52,53,56]$, and in the current study, we observed that higher levels of dementia symptomology (as measured by the DLD) were associated with greater ventricular volume, an MRI biomarker of neurodegeneration.

$A P O E$ is a very strong genetic risk factor predisposing patients to $\mathrm{AD}$-associated neurodegeneration; TD adults who are carriers of the $\varepsilon 4$ risk allele show more signs of neurodegeneration before symptom onset [57]. While $A P O E$ is also known to be associated with further increased risk of AD in the DS population, to our knowledge, this is the first study in DS to investigate the association of APOE \&4 carrier status with MRI volume data [39]. The observed $\varepsilon 4$ carrier frequency was $3 / 28$ alleles, or $11 \%$, which is similar to the frequency observed in the general population (13\%). However, one of the three $\varepsilon 4$ carriers was the DS group outlier, and it was this subject whose data drove the observed effect on brain volume by $A P O E$ genotype. Thus, we are not able to make a strong conclusion based on these data, and future analyses with larger sample sizes will be necessary to confirm an effect of $A P O E$ on age-related differences in brain volume in DS.

The present results must be interpreted within the framework of our statistical models. The WS and DS groups differed in mean age, but in all cases, we included age and sex as important covariates known to be related to neurodegeneration. The highly significant $P$-values we observed seem to be driven in part by one DS outlier, but the trends did not change when the outliers were removed (Additional file 1: Figure S1). Furthermore, though this study found a significant agerelated difference in $\mathrm{DS}$ adults in $\mathrm{AD}$-related regions, we may have been underpowered to detect more subtle AD-related changes due to 1 ) the relatively small sample size of the DS group, 2) the strict statistical threshold used for significance, and 3) the fact that FreeSurfer parameter estimates for smaller subcortical areas, such as the entorhinal cortex and hippocampus, are known to exhibit greater error [58]. Despite this, some ADrelated regions were nominally significant at an unadjusted $P<.05$ (left hippocampus: $P=.0023$, right amygdala: $P=.0042$, left amygdala: $P=.0042$, right posterior cingulate gyrus: $P=.0279$, left posterior cingulate gyrus: $P=.0495)$. These results warrant further analysis of age-related affects in AD-related regions in a larger cohort of DS adults.

\section{Conclusion}

In conclusion, individuals with DS demonstrated a unique pattern of age-related effects on gray matter and ventricular volume, the latter of which was associated with dementia rating scores in the DS group. Results may indicate that early onset of brain aging in DS is primarily due to DS-specific neurodegenerative processes, as opposed to general atypical neurodevelopment.

\section{Additional file}

\begin{abstract}
Additional file 1: Table S1a. Regions of interest included in analysis. Table S1b. Regions of interest not included in analysis. Table S2. Additional demographics for Down syndrome participants, including APOE \&4 genotype, test scores for the Dementia Questionnaire for People with Learning Disabilities (DLD) (sum of cognitive scores (SCS) and sum of social scores (SOS)), and the Kaufman Brief Intelligence Test (KBIT) composite score. Table S3. complete results from analysis of relationship between volume and age comparing the Down syndrome (DS) and Williams syndrome (WS) groups to the typically developing (TD) controls. Table S4. Results from analysis comparing relationship between volume and age between participants with Down syndrome and Williams syndrome. Table S5. To investigate whether the observed effects were being driven by outliers, we removed the subjects whose ICV-corrected total gray matter volume was outside of two standard deviations of the grand mean and re-ran our analyses. Figure S1. This figure shows results from post hoc analyses which excluded subjects whose ICV-normalized gray matter volumes fell outside two standard deviations of the grand mean for their respective group (one with DS and four with WS). Figure S2. regional brain volumes normalized to ICV are plotted in relationship to Dementia Questionnaire for People with Learning Disabilities (DLD) sum of cognitive scores (SCS) across the Down syndrome subject group in: (a) right inferior lateral ventricle (RILV) and (b) left inferior lateral ventricle (LILV). Figure S3. Regional brain volumes normalized to ICV are plotted in relationship to Dementia Questionnaire for People with Learning Disabilities (DLD) sum of social scores (SOS) across the Down syndrome subject group in: (a) right inferior lateral ventricle (RILV) and (b) left inferior lateral ventricle (LILV). Figure S4. While APOE ع4 carrier status showed a trend for association with regional brain volume, a larger sample size will be needed to accurately estimate this effect.
\end{abstract}

\section{Abbreviations}

AD: Alzheimer's disease; APOE: apolipoprotein-E gene; DLD: Dementia Questionnaire for People with Learning Disabilities; DLD-SOS: Sum of Social Scores for DLD; DLD-SCS: Sum of Cognitive Scores for DLD; DS: Down syndrome; ICV: (total) intracranial volume; LILV: left inferior lateral ventricle; $\mathrm{MCl}$ : mild cognitive impairment; MRI: magnetic resonance imaging; PET: positron emission tomography; RILV: right inferior lateral ventricle; ROI: (brain) region of interest; SNP: single nucleotide polymorphism; TD: typical development; WS: Williams syndrome.

\section{Competing interests}

The authors declare that they have no competing interests. 


\section{Authors' contributions}

MEK contributed to study design, carried out the statistical analyses, participated in subject recruitment, scanning, imaging analysis and quality control, and drafted the manuscript. CME contributed to imaging analysis and quality control and helped to draft the manuscript. TJH contributed to study design, participated in its coordination, and helped to draft the manuscript. JNV and JP participated in subject recruitment, scanning, and imaging analysis. LES, GC, and LVR contributed to participant recruitment, neuropsychological testing, and genotyping. SAM participated in scanning and imaging analysis. ND contributed to imaging analysis and quality control. SNA and JUB participated in subject recruitment and scanning and helped to draft the manuscript. EMD participated in the study design and helped to draft the manuscript. TATW conceived of the study, participated in its design and the coordination of subject recruitment, scanning and imaging quality control, and helped to draft the manuscript. All authors read and approved the final manuscript.

\section{Acknowledgments}

We would like to thank all the study volunteers for their time and willingness to participate in this research. We are grateful for the sponsors of the Williams Syndrome Music Camp, including the Vanderbilt Kennedy Center for Research on Human Development, the Academy of Country Music's Lifting Lives program, and the Vanderbilt Blair School of Music. We would also like to thank and acknowledge the research staff of the Williams Syndrome Music Camp, especially Elizabeth Roof, and the MRI technologists in the Vanderbilt University Institute of Imaging Science, including Dave Pennell, Leslie McIntosh, Kristen George-Durrett, and Donna Butler, for their expertise, professionalism, and wonderful way with participants. This research was supported in large part by a Vanderbilt Kennedy Center Hobbs Discovery Grant (to TATW). Additional support was by the Recruitment for Genetic Aging Research (P30 AG036445 to TATW), the Vanderbilt/National Institute of Mental Health Neurogenomics Training grant (T32 MH65215 to TJH), a PhRMA Foundation Postdoctoral fellowship in Translational Medicine and Therapeutics (to TJH), the Public Health Service award from the National Institute of General Medical Studies for the Vanderbilt Medical-Scientist Training Program (T32 GM07347 to MEK), the Neuroimaging and Genetic Study of Inhibited Temperament (K01-MH083052 to JUB), and the CTSA award UL1TR000445 from the National Center for Advancing Translational Sciences. The funders had no role in study design, data collection and analysis, decision to publish, or preparation of the manuscript. Its contents are solely the responsibility of the authors and do not necessarily represent official views of the National Center for Advancing Translational Sciences or the National Institutes of Health.

\section{Author details}

${ }^{1}$ Center for Human Genetics and Research, Department of Molecular Physiology \& Biophysics, Vanderbilt University School of Medicine, 37232-0700, 519 Light Hall, Nashville, TN, USA. ${ }^{2}$ Medical Scientist Training Program, Vanderbilt University School of Medicine, Nashville, TN, USA. ${ }^{3}$ Short-Term Training Program Undergraduate Research Fellow, Vanderbilt University, Nashville, TN, USA. ${ }^{4}$ Neuroscience Graduate Program, Vanderbilt University, Nashville, TN, USA. ${ }^{5}$ Interdisciplinary Studies in Neuroimaging of Neurodevelopmental Disorders, The Graduate School, Vanderbilt University, Nashville, USA. ${ }^{6}$ Graduate Program in Clinical Psychological Sciences, Department of Psychology, Vanderbilt University, Nashville, TN, USA. 7 Department of Psychiatry, Vanderbilt University School of Medicine, Nashville, TN, USA. ${ }^{8}$ Vanderbilt Kennedy Center for Research on Human Development, Vanderbilt University, Nashville, TN, USA. 'Department of Psychology and Human Development, Vanderbilt University, Nashville, TN, USA. ${ }^{10}$ Vanderbilt University Institute of Imaging Science, Vanderbilt University, Nashville, TN, USA.

Received: 19 September 2013 Accepted: 20 March 2014 Published: 9 April 2014

\section{References}

1. Beacher F, Daly E, Simmons A, Prasher V, Morris R, Robinson C, Lovestone S, Murphy K, Murphy DGM: Brain anatomy and ageing in non-demented adults with Down's syndrome: an in vivo MRI study. Psychol Med 2010, 40:611-619.

2. Parker SE, Mai CT, Canfield MA, Rickard R, Wang Y, Meyer RE, Anderson P, Mason CA, Collins JS, Kirby RS, Correa A: Updated National Birth
Prevalence estimates for selected birth defects in the United States, 2004 to 2006. Birth Defects Res A Clin Mol Teratol 2010, 88:1008-1016.

3. Mégarbané A, Ravel A, Mircher C, Sturtz F, Grattau Y, Rethoré M-O, Delabar J-M, Mobley WC: The 50th anniversary of the discovery of trisomy 21: the past, present, and future of research and treatment of Down syndrome. Genet Med 2009, 11:611-616.

4. Lott IT, Dierssen M: Cognitive deficits and associated neurological complications in individuals with Down's syndrome. Lancet Neurol 2010, 9:623-633.

5. Dierssen M: Down syndrome: the brain in trisomic mode. Nat Rev Neurosci 2012, 13:844-858

6. Penrose $\mathrm{L}$ : The incidence of mongolism in the general population. $J$ Ment Sci 1949, 95:685-688

7. Urbano RC: (Ed): International Review of Research in Mental Retardation: Health Issues in Down Syndrom. San Diego, CA: Academic Press; 2010.

8. Korenberg JR, Chen XN, Hirota H, Lai Z, Bellugi U, Burian D, Roe B, Matsuoka R: VI Genome structure and cognitive map of Williams syndrome. J Cogn Neurosci 2000, 12(Suppl):89-107.

9. Strømme P, Bjørnstad PG, Ramstad K: Prevalence estimation of Williams syndrome. J Child Neurol 2002, 17:269-271.

10. Martens MA, Wilson SJ, Reutens DC: Research review: Williams syndrome: a critical review of the cognitive, behavioral, and neuroanatomical phenotype. J Child Psychol Psychiatry 2008, 49:576-608.

11. Meyer-Lindenberg A, Mervis CB, Berman KF: Neural mechanisms in Williams syndrome: a unique window to genetic influences on cognition and behaviour. Nat Rev Neurosci 2006, 7:380-393.

12. Conners FA, Moore MS, Loveall SJ, Merrill EC: Memory profiles of Down, Williams, and fragile $X$ syndromes: implications for reading development. J Dev Behav Pediatr 2011, 32:405-417.

13. Dykens EM, Rosner BA, Ly T, Sagun J: Music and anxiety in Williams syndrome: a harmonious or discordant relationship? Am J Ment Retard 2005, 110:346-358.

14. Hocking DR, Rinehart NJ, McGinley JL, Moss SA, Bradshaw JL: A kinematic analysis of visually-guided movement in Williams syndrome. J Neurol SCi 2011, 301:51-58.

15. Mobbs D, Eckert MA, Mills D, Korenberg J, Bellugi U, Galaburda AM, Reiss AL: Frontostriatal dysfunction during response inhibition in Williams syndrome. Biol Psychiatry 2007, 62:256-261.

16. Tsai S-W, Wu S-K, Liou Y-M, Shu S-G: Early development in Williams syndrome. Pediatr Int 2008, 50:221-224.

17. Dykens EM: Anxiety, fears, and phobias in persons with Williams syndrome. Dev Neuropsychol 2003, 23:291-316.

18. Mervis CB, John AE: Cognitive and behavioral characteristics of children with Williams syndrome: implications for intervention approaches. Am J Med Genet C: Semin Med Genet 2010, 154C:229-248.

19. Morris CA: The behavioral phenotype of Williams syndrome: a recognizable pattern of neurodevelopment. Am J Med Genet C: Semin Med Genet 2010, 154C:427-431

20. Cherniske EM, Carpenter TO, Klaiman C, Young E, Bregman J, Insogna K, Schultz RT, Pober BR: Multisystem study of 20 older adults with Williams syndrome. Am J Med Genet A 2004, 131:255-264.

21. Reiss AL, Eliez S, Schmitt JE, Straus E, Lai Z, Jones W, Bellugi U: IV. Neuroanatomy of Williams syndrome: a high-resolution MRI study. J Cogn Neurosci 2000, 12(1):65-73. 0898-929X (Print).

22. Chiang M-C, Reiss AL, Lee AD, Bellugi U, Galaburda AM, Korenberg JR, Mills DL, Toga AW, Thompson PM: 3D pattern of brain abnormalities in Williams syndrome visualized using tensor-based morphometry. Neuroimage 2007, 36:1096-1109.

23. Meda SA, Pryweller JR, Thornton-Wells TA: Regional brain differences in cortical thickness. Surface area and subcortical volume in individuals with Williams syndrome. PLoS One 2012, 7:e31913.

24. Jernigan TLL, Bellugi U, Sowell E, Doherty S, Hesselink JRR: Cerebral morphologic distinctions between Williams and Down syndromes. Arch Neurol 1993, 50:186-191.

25. Teipel SJ, Alexander GE, Schapiro MB, Moller HJ, Rapoport SI, Hampel H: Age-related cortical grey matter reductions in non-demented Down's syndrome adults determined by MRI with voxel-based morphometry. Brain 2004, 127(Pt 4):811-824

26. Devenny DA, Krinsky-McHale SJ, Kittler PM, Flory M, Jenkins E, Brown WT: Developmental neuropsychology Age-associated memory changes in adults with Williams syndrome. Dev Neuropsychol 2004, 26:691-706. 
27. Dale A, Fischl B, Sereno MI: Cortical surface-based analysis: I. Segmentation and Surface Reconstruction. Neuroimage 1999, 9:179-194.

28. Segonne F, Dale AM, Busa E, Glessner M, Salat D, Hahn HK, Fischl B: A hybrid approach to the skull stripping problem in MRI. Neuroimage 2004, 22:1060-1075

29. Fischl B, van der Kouwe A, Destrieux C, Halgren E, Ségonne F, Salat DH, Busa E, Seidman $\sqcup$, Goldstein J, Kennedy D, Caviness V, Makris N, Rosen B, Dale AM: Automatically parcellating the human cerebral cortex. Cereb Cortex 2004, 14:11-22.

30. Segonne F, Pacheco J, Fischl B: Geometrically accurate topologycorrection of cortical surfaces using nonseparating loops. IEEE Trans Med Imaging 2007, 26:518-529.

31. Desikan RS, Ségonne F, Fischl B, Quinn BT, Dickerson BC, Blacker D, Buckne RL, Dale AM, Maguire RP, Hyman BT, Albert MS, Killiany RJ: An automated labeling system for subdividing the human cerebral cortex on MRI scans into gyral based regions of interest. Neuroimage 2006, 31:968-980.

32. Evenhuis HM: Further evaluation of the Dementia Questionnaire for Persons with Mental Retardation (DMR). J Intellect Disab Res 1996, 40:369-373.

33. Prasher VP: Dementia Questionnaire for Persons with Mental Retardation (DMR): Modified criteria for adults with Down's syndrome. J Appl Res Intellect Disabil 1997, 10:54-60.

34. Shultz J, Aman M, Kelbley T, LeClear Wallace C, Burt DB, Primeaux-Hart S, Loveland K, Thorpe L, Bogos ES, Timon J, Patti P, Tsiouris J: Evaluation of screening tools for dementia in older adults with mental retardation. Am J Ment Retard 2004, 109:98-110.

35. Coding of categorical predictors and ANCOVA. [http://www.upa.pdx.edu/ IOA/newsom/da2/ho_coding1.pdf]

36. Evenhuis HM: The natural history of dementia in Down's syndrome. Arch Neurol 1990, 47:263-267.

37. Zigman WB, Schupf N, Devenny DA, Miezejeski C, Ryan R, Un TK, Schubert R, Silverman W: Incidence and prevalence of dementia in elderly adults with mental retardation without Down syndrome. Am J Ment Retard 2004, 109:126-141.

38. Oyama F, Cairns NJ, Shimada H, Oyama R, Titani K, Ihara Y: Down's Syndrome: up-regulation of $\beta$-amyloid protein precursor and $\mathrm{\tau}$ mRNAs and their defective coordination. J Neurochem 1994, 62:1062-1066.

39. Coppus AMW, Evenhuis HM, Verberne G-J, Visser FE, Arias-Vasquez A, Sayed-Tabatabaei FA, Vergeer-Drop J, Eikelenboom P, van Gool WA, van Duijn CM: The impact of apolipoprotein $\mathrm{E}$ on dementia in persons with Down's syndrome. Neurobiol Aging 2008, 29:828-835.

40. Prasher VP, Farrer MJ, Kessling AM, Fisher EM, West RJ, Barber PC, Butler AC Molecular mapping of Alzheimer-type dementia in Down's syndrome. Ann Neurol 1998, 43:380-383.

41. Mann DM: The pathological association between Down syndrome and Alzheimer disease. Mech Ageing Dev 1988, 43:99-136.

42. Storandt M, Mintun MA, Head D, Morris JC: Cognitive decline and brain volume loss as signatures of cerebral amyloid-beta peptide deposition identified with Pittsburgh compound B: cognitive decline associated with Abeta deposition. Arch Neurol 2009, 66:1476-1481.

43. Handen BL, Cohen AD, Channamalappa U, Bulova P, Cannon SA, Cohen WI, Mathis CA, Price JC, Klunk WE: Imaging brain amyloid in nondemented young adults with Down syndrome using Pittsburgh compound B. Alzheimers Dement 2012, 8:496-501.

44. Thompson PM, Hayashi KM, de Zubicaray GI, Janke AL, Rose SE, Semple J, Hong MS, Herman DH, Gravano D, Doddrell DM, Toga AW: Mapping hippocampal and ventricular change in Alzheimer disease. Neuroimage 2004, 22:1754-1766.

45. Jack CR, Slomkowski M, Gracon S, Hoover TM, Felmlee JP, Stewart K, Xu Y, Shiung M, O'Brien PC, Cha R, Knopman D, Petersen RC: MRI as a biomarker of disease progression in a therapeutic trial of milameline for $A D$. Neurology 2003, 60:253-260.

46. Ridha BH, Anderson VM, Barnes J, Boyes RG, Price SL, Rossor MN, Whitwell $J$, Jenkins L, Black RS, Grundman M, Fox NC: Volumetric MRI and cognitive measures in Alzheimer disease : comparison of markers of progression. J Neurol 2008, 255:567-574.

47. Jack CR, Shiung MM, Gunter JL, O'Brien PC, Weigand SD, Knopman DS, Boeve BF, Ivnik RJ, Smith GE, Cha RH, Tangalos EG, Petersen RC: Comparison of different MRI brain atrophy rate measures with clinical disease progression in AD. Neurology 2004, 62:591-600.

48. Weiner MW: Expanding ventricles may detect preclinical Alzheimer disease. Neurology 2008, 70:824-825.
49. Minati L, Edginton T, Bruzzone MG, Giaccone G: Current concepts in Alzheimer's disease: a multidisciplinary review. Am J Alzheimers Dis Other Demen 2009, 24:95-121.

50. Haier RJ, Head K, Head E, Lott IT: Neuroimaging of individuals with Down's syndrome at-risk for dementia: evidence for possible compensatory events. Neuroimage 2008, 39:1324-1332.

51. Kesslak JP, Nagata SF, Lott I, Nalcioglu O: Magnetic resonance imaging analysis of age-related changes in the brains of individuals with Down's syndrome. Neurology 1994, 44:1039-1045.

52. Pearlson GDD, Breiter SNN, Aylward EHH, Warren ACC, Grygorcewicz M, Frangou S, Barta PEE, Pulsifer MBB: MRI brain changes in subjects with Down syndrome with and without dementia. Dev Med Child Neurol 1998, 40:326-334.

53. Pinter JD, Brown WE, Eliez S, Schmitt JE, Capone GT, Reiss AL: Amygdala and hippocampal volumes in children with Down syndrome: a high-resolution MRI study. Neurology 2001, 56:972-974.

54. Mann DM, Esiri MM: The pattern of acquisition of plaques and tangles in the brains of patients under 50 years of age with Down's syndrome. J Neurol Sci 1989, 89:169-179.

55. Chou Y-Y, Leporé N, Chiang M-C, Avedissian C, Barysheva M, McMahon KL, de Zubicaray Gl, Meredith M, Wright MJ, Toga AW, Thompson PM: Mapping genetic influences on ventricular structure in twins. Neuroimage 2009, 44:1312-1323

56. Aylward EH, Li Q, Honeycutt NA, Warren AC, Pulsifer MB, Barta PE, Chan MD, Smith PD, Jerram M, Pearlson GD: MRI volumes of the hippocampus and amygdala in adults with Down's syndrome with and without dementia. Am J Psychiatry 1999, 156:564-568.

57. Wishart HA, Saykin AJ, McAllister TW, Rabin LA, McDonald BC, Flashman LA, Roth RM, Mamourian AC, Tsongalis GJ, Rhodes CH: Regional brain atrophy in cognitively intact adults with a single APOE epsilon4 allele. Neurology 2006, 67:1221-1224

58. Cherbuin N, Anstey KJ, Réglade-Meslin C, Sachdev PS: In vivo hippocampal measurement and memory: a comparison of manual tracing and automated segmentation in a large community-based sample. PLoS One 2009, 4:e5265.

doi:10.1186/1866-1955-6-8

Cite this article as: Koran et al:: Differences in age-related effects on brain volume in Down syndrome as compared to Williams syndrome and typical development. Journal of Neurodevelopmental Disorders 2014 6:8

\section{Submit your next manuscript to BioMed Central and take full advantage of:}

- Convenient online submission

- Thorough peer review

- No space constraints or color figure charges

- Immediate publication on acceptance

- Inclusion in PubMed, CAS, Scopus and Google Scholar

- Research which is freely available for redistribution 